\title{
Conductas de exposición y protección solar en adolescentes y adultos de la Ciudad de México
}

\author{
Martha A. Morales-Sánchez, * Frida D. Navarro-Rodríguez, Ma. Guadalupe Olguín-García, \\ Myrna Rodríguez-Acar, Ma. Luisa Peralta-Pedrero y Fermín Jurado-Santa Cruz \\ Centro Dermatológico Dr. Ladislao de la Pascua, Servicios de Salud Pública, Ciudad de México, México
}

\section{Resumen}

Objetivo: Conocer la prevalencia de las conductas exposición y protección solar en un grupo de adolescentes y compararlos con un grupo de adultos en la Ciudad de México. Material y métodos: Estudio transversal descriptivo en estudiantes de 16 a 18 años y en adultos mayores de 18 años. Resultados: Se encuestaron 748 alumnos, $60 \%$ mujeres y $40 \%$ hombres. El $90 \%$ busca la sombra, el $70.1 \%$ evita exponerse al sol de las 10 a las 16 horas y el $30.6 \%$ usa filtro solar. De 620 adultos, $67.5 \%$ mujeres y $32.4 \%$ hombres, el $82.6 \%$ buscan la sombra, el $60.2 \%$ evita el sol de las 10 a las 16 horas y el $48.1 \%$ utiliza filtro solar. El $72.9 \%$ de adultos y el $80.8 \%$ de adolescentes se exponen al sol debido a actividades domésticas. Discusión: Existe una tendencia similar en las conductas de protección y exposición solar en adolescentes y adultos. Es alta la prevalencia de uso de fotoprotector en adultos en comparación con los adolescentes.

PALABRAS CLAVE: Conductas de exposición solar. Conductas de protección solar. Fotoprotección. Prevención del cáncer de piel.

\section{Sun exposure and sun protection behaviors among teenagers and adults in Mexico City}

\begin{abstract}
Objective: To know the prevalence of sun exposure and protection behaviors in a group of adolescents and to compare them with a group of adults in Mexico City. Material and methods: Descriptive, cross-sectional study in students aged 16 to 18 years and in adults older than 18 years. Results: Seven-hundred and forty-eight students were surveyed; $60 \%$ were females and $40 \%$ males; $90 \%$ seek shade, $70.1 \%$ avoid exposing themselves to the sun from 10.00 to 16.00 hours and $30.6 \%$ use sunscreen. Out of 620 adults, $67.5 \%$ were women and $32.4 \%$ were men; $82.6 \%$ seek shade, $60.2 \%$ avoid the sun from 10.00 to 16.00 hours and $48.1 \%$ use sunscreen; $72.9 \%$ of adults and $80.8 \%$ of adolescents are exposed to the sun due to domestic activities. Discussion: There is a similar trend in sun protection and exposure behaviors in adolescents and adults. The prevalence of sunscreen use in adults is high in comparison with adolescents.
\end{abstract}

KEY WORDS: Sun exposure behaviors. Sun protection behaviors. Photoprotection. Skin cancer prevention. 


\section{Introducción}

La exposición crónica a la radiación ultravioleta es el factor de riesgo más importante para cáncer cutáneo, principalmente queratinocítico y melanoma. ${ }^{1}$ En México, el cáncer de piel ocupa el segundo lugar entre las neoplasias malignas más frecuentes (13.6\%) y el $87.2 \%$ de los casos reportados desarrollaron el tumor en piel descubierta y expuesta al sol, como la cara y extremidades superiores. ${ }^{2,3}$

La Organización Mundial de la Salud (OMS) define como conducta de riesgo a toda aquella exposición de un individuo que aumente su probabilidad de sufrir una enfermedad o lesión. ${ }^{4}$ En el caso del cáncer de piel, las conductas de riesgo se relacionan con la exposición aguda o crónica a la radiación ultravioleta. Las recomendaciones para evitar el daño cutáneo secundario a exposición solar son: evitar la exposición entre las 10 y las 16 horas; usar ropa de colores oscuros, tejidos sintéticos de fibras cerradas, manga larga; buscar la sombra; utilizar sombrero o sombrilla y aplicarse diariamente un filtro solar. ${ }^{5}$

Para medir las conductas de exposición solar pueden utilizarse métodos directos e indirectos. Los primeros miden la exposición a la radiación ultravioleta con un dosímetro portátil, mientras que los segundos miden dicha exposición mediante la aplicación de cuestionarios autoadministrados.

Aunque se han realizado estudios sobre las conductas de exposición y protección relacionadas al sol, en los últimos años no se ha medido la prevalencia de estos hábitos en la población de la Ciudad de México. Por lo que el objetivo de este estudio fue determinar la prevalencia de las conductas exposición y de protección solar en un grupo de adolescentes y compararlos con un grupo de adultos que habitan en la Ciudad de México.

\section{Material y métodos}

Se realizó un estudio transversal o de encuesta entre estudiantes de preparatoria y adultos que acudieron al Centro Dermatológico Dr. Ladislao de la Pascua (CDP) de la Ciudad de México. El estudio se realizó en dos etapas: 1) validación del cuestionario autoaplicado, y 2) aplicación del cuestionario a las poblaciones objetivo. El protocolo de investigación fue aprobado por el Comité de Investigación y de Ética en Investigación de la institución.

\section{Validación del cuestionario autoaplicado CONRESOL}

Se realizó una revisión sistemática sobre los cuestionarios autoaplicados para medir la exposición solar, posteriormente se identificaron las dimensiones del constructo a medir: "exposición" y "protección". A partir de la investigación bibliográfica y con los resultados de una encuesta realizada a 101 participantes (con la técnica de preguntas abiertas para explorar la terminología y el contenido del constructo) se construyó el cuestionario CONRESOL. La primera versión del cuestionario fue revisada por un grupo de ocho expertos, para la validación de apariencia y contenido de los ítems, dando lugar a una segunda versión. Posteriormente se realizaron tres pruebas piloto con la finalidad de evaluar las escalas de respuesta por ítem, la comprensión del vocabulario y el tiempo promedio de respuesta. Para la validación empírica se utilizó la 5. ${ }^{a}$ versión del cuestionario, la cual se contestaba en un promedio de 10 minutos y se aplicó a 350 participantes. Este tamaño de muestra se calculó por el número de ítems propuestos inicialmente. Se eliminaron los ítems con una correlación total menor a 0.3 , correlación múltiple al cuadrado menor a 0.40 y correlación alta entre ellos ( $\geq 0.8)$, seleccionando al ítem con el mejor perfil. Posterior a esta reducción de ítems, se realizó un análisis factorial para la validación de constructo, excluyendo los coeficientes menores a 0.40 de acuerdo con el porcentaje de varianza explicado y el punto de quiebre en la gráfica de sedimentación. Al final se identificó una dimensión denominada "conductas de protección solar" y otra de "conductas de exposición solar" con tres indicadores. El cuestionario final quedó conformado por 13 ítems, distribuidos en dos dimensiones: la primera con una escala en la que se preguntan los días de la semana en que se realizan dichas conductas y la segunda con una escala de respuesta tipo Likert. La consistencia interna del instrumento fue de 0.865 de acuerdo con el cálculo del alfa de Cronbach.

\section{Aplicación del cuestionario a las poblaciones objetivo}

Los cuestionarios se aplicaron a dos poblaciones: a) estudiantes de la Escuela Nacional Preparatoria Vidal Castañeda y Nájera de la Universidad Nacional Autónoma de México, y b) adultos que acudieron al CDP. Se midieron las variables demográficas y las 
relativas a las conductas de protección solar y exposición solar con el cuestionario validado exprofeso para este estudio. El CONRESOL interroga sobre los hábitos relacionados al sol de una semana habitual durante el último mes y no es sensible a cambios de conductas a lo largo del año, ni proporciona información sobre un periodo de tiempo de la vida del encuestado.

\section{Tamaño de muestra}

Para el cálculo para la validación empírica del cuestionario CONRESOL se utilizó la fórmula $n=k(10)$, en donde $\mathrm{k}$ es el número de ítems del instrumento preliminar. Para calcular el tamaño de muestra mínimo de las poblaciones objetivo se utilizó la fórmula para la estimación de una proporción, considerando que al menos el $30 \%$ de los participantes realizarían al menos alguna actividad de protección solar, de acuerdo con lo reportado en estudios previos en otras poblaciones. Para dicho cálculo se consideró un alfa de 0.05 y una precisión del $3 \%$.

\section{Análisis estadístico}

La captura, procesamiento y análisis estadístico de los datos obtenidos se realizó con el programa SPSS versión 19.0. Para el análisis descriptivo de las variables cualitativas se calcularon proporciones y para las variables cuantitativas con distribución normal media y desviación estándar. Posteriormente se compararon las prevalencias de las conductas relacionadas al sol de las dos poblaciones estudiadas con la prueba de $\chi^{2}$, considerando significancia estadística al obtener una $p<0.05$.

\section{Resultados}

\section{Características de la muestra de validación}

Las características sociodemográficas de la muestra para validación $(\mathrm{n}=350)$ son las siguientes: $68.9 \%$ fueron mujeres $(n=241)$ y el $31.1 \%$ fueron hombres ( $n=109)$; el $50 \%$ de la muestra tenía de 18 a 37 años de edad (rango intercuartílico de 24 años); la escolaridad máxima fue licenciatura, en el $35.4 \%$. La clasificación por ocupación fue: $23.3 \%$ amas de casa, $20.3 \%$ estudiantes, $14 \%$ profesionistas, $14 \%$ empleados administrativos, $14 \%$ trabajadores de
Tabla 1. Conductas de protección solar en adolescentes y adultos

\begin{tabular}{|c|c|c|c|}
\hline & Conductas de protección solar & $\begin{array}{l}\text { Preparatoria } \\
n=748(\%)\end{array}$ & $\begin{array}{l}\text { CDP } \\
n=620(\%)\end{array}$ \\
\hline 1 & $\begin{array}{l}\text { Usar protector solar, FPS }>30 \text {, } \\
\text { cada } 2 \text { a } 4 \text { horas }\end{array}$ & $229(30.6)$ & $298(48.1)^{\star}$ \\
\hline 2 & $\begin{array}{l}\text { Buscar la sombra para evitar } \\
\text { el sol }\end{array}$ & $673(90)$ & $512(82.6)^{\star}$ \\
\hline 3 & Usar sombrilla & $77(10.3)$ & $162(26.2)^{*}$ \\
\hline 4 & Usar sombrero & $111(14.8)$ & $278(44.8)^{*}$ \\
\hline 5 & Usar lentes de sol & $222(29.7)$ & $205(33.1)^{*}$ \\
\hline 6 & $\begin{array}{l}\text { Evitar el sol de las 10:00 a las } \\
16: 00 \mathrm{~h}\end{array}$ & $524(70.1)$ & $373(60.2)^{\star}$ \\
\hline
\end{tabular}

servicios y $14.4 \%$ comerciantes o vendedores. El $65.1 \%$ de los encuestados vivían en la Ciudad de México, el $20.6 \%$ en el Estado de México y el $1.7 \%$ en Hidalgo.

\section{Prevalencia de las conductas de protección y riesgo}

\section{Adolescentes}

Se encuestaron 748 alumnos, de los cuales el $60 \%$ $(n=449)$ fueron mujeres y el $40 \%(n=299)$ hombres. El $40.5 \%$ de los estudiantes tuvieron una media de edad de 16 años. En cuanto a la prevalencia de las conductas de protección solar, la que con mayor frecuencia realizan es el buscar la sombra en días soleados, con el $90 \%(n=673)$, mientras que la de menor frecuencia es usar sombrilla, con solo el $10.3 \%$ $(n=77)$. Del total del encuestados, el $80.8 \%(n=605)$ refirió exponerse al sol al realizar actividades domésticas como lavar ropa o limpiar el patio. Por otro lado, la conducta de exposición solar con menor prevalencia fue conducir un vehículo, un $23.8 \%$ ( $n=178)$. El resto de las prevalencias de las conductas de protección y riesgo se muestran en las Tablas 1 y 2 .

\section{Adultos}

Se encuestaron 620 pacientes, 419 mujeres y 201 hombres, que corresponde al $67.5 \%$ y $32.4 \%$ respectivamente, con una mediana de 39 años de edad y un rango de 18 a 60 años. Para el $40.3 \%(n=250)$ de los pacientes era su primera consulta, mientras 
Tabla 2. Conductas de exposición solar en adolescentes y adultos

\begin{tabular}{|l|l|l|c|}
\hline & $\begin{array}{l}\text { Conductas de exposición } \\
\text { solar }\end{array}$ & $\begin{array}{c}\text { Preparatoria } \\
n=748(\%)\end{array}$ & $\begin{array}{c}\text { CDP } \\
n=620(\%)\end{array}$ \\
\hline 7 & Tomar el sol & $545(72.8)$ & $238(38.4)^{*}$ \\
\hline 8 & $\begin{array}{l}\text { Realizar actividades deportivas } \\
\text { al aire libre }\end{array}$ & $572(76.5)$ & $288(46.4)^{*}$ \\
\hline 9 & Trabajar en exteriores & $444(59.3)$ & $252(40.6)^{*}$ \\
\hline 10 & Conducir un vehículo & $178(23.8)$ & $212(34.2)^{*}$ \\
\hline 11 & Conducir bicicleta & $292(39.0)$ & $132(21.3)^{*}$ \\
\hline 12 & Ir a la playa & $262(35.0)$ & $191(30.8)^{*}$ \\
\hline 13 & $\begin{array}{l}\text { Realizar actividades domésticas } \\
\text { al aire libre (tender ropa, barrer } \\
\text { el patio, lavar) }\end{array}$ & $605(80.8)$ & $452(72.9)^{*}$ \\
\hline $\begin{array}{l}\text { CDP }=\text { Centro Dermatológico Dr. Ladislao de la Pascua. } \\
\text { *Chi cuadrada, } p<0.001 .\end{array}$ & & \\
\hline
\end{tabular}

que el $59.6 \%(n=370)$ eran subsecuentes. La ocupación más común fue ama de casa, en un $29.8 \%$, y la escolaridad predominante de los pacientes fue bachillerato, con un $40.6 \%$.

Las conductas de protección solar que los pacientes refirieron realizar con mayor frecuencia fueron buscar la sombra, un $82.6 \%(n=512)$, y evitar el sol de las 10 a las 16 horas, el $60.2 \%(n=373)$. La medida de protección realizada con menor frecuencia fue el uso de sombrilla, con el $26.2 \%$ ( $n=162)$. En este grupo, la principal causa de exposición al sol fueron las actividades domésticas al aire libre, con el $72.9 \%$ ( $n=452$ ), seguida de las actividades deportivas en exteriores, con el $46.4 \%(n=288)$ (Tablas 1 y 2$)$.

La mediana de tiempo de exposición solar en un día habitual fue de 2.4 horas ( \pm 3 horas). Se interrogó sobre el conocimiento del índice de radiación ultravioleta; únicamente el $3.4 \%$ afirmó conocerlo y $1.9 \%$ lo consulta diariamente.

Al comparar las prevalencias de las conductas de protección solar y riesgo entre ambas poblaciones objetivo, encontramos diferencias estadísticamente significativas $(p<0.001)$ para todas las conductas. Dentro de las de protección, en las que se documentó una diferencia de al menos el doble, fue para el uso de sombrilla y el uso de sombrero; ambas conductas fueron más frecuentes en los adultos que en los adolescentes, 26.2 versus $10.3 \%$ y 44.8 versus $14.8 \%$ respectivamente (Tabla 1). Sin embargo, las mayores diferencias se registraron en las conductas de exposición solar, en donde tomar el sol y realizar actividades deportivas al aire libre son más prevalentes en
Tabla 3. Exposición solar durante los fines de semana en adolescentes y adultos

\begin{tabular}{l|c|c|c|}
$\begin{array}{l}\text { Conductas de } \\
\text { exposición solar }\end{array}$ & $\begin{array}{c}\text { Preparatoria } \\
n=748(\%)\end{array}$ & $\begin{array}{c}\text { CDP } \\
n=620(\%)\end{array}$ & Total \\
\hline $\begin{array}{l}\text { Se exponen al sol en } \\
\text { fines de semana }\end{array}$ & $327(43.8)$ & $331(53.4)^{\star}$ & $658(48.1)$ \\
\hline $\begin{array}{l}\text { Evitan el sol en fines } \\
\text { de semana }\end{array}$ & $420(56.2)$ & $289(46.6)^{\star}$ & $709(51.9 \%)$ \\
$\begin{array}{l}\text { CDP = Centro Dermatológico Dr. Ladislao de la Pascua. } \\
\text { *Chi cuadrada, } p<0.001 .\end{array}$ & & \\
\end{tabular}

los estudiantes de preparatoria en comparación con los pacientes del CDP (Tabla 2). Los pacientes subsecuentes utilizan con mayor frecuencia filtro solar en comparación con los pacientes atendidos por primera vez, 57.8 y $34.1 \%$ respectivamente $(p<0.0001)$. El $43.8 \%(n=327)$ de los estudiantes y el $53.4 \%$ ( $n=331)$ de los adultos se exponen más al sol los fines de semana que de lunes a viernes (Tabla 3 ).

\section{Discusión}

Existe una tendencia similar entre los adolescentes y adultos en relación con las conductas de exposición solar, tanto de riesgo como de protección. Ambos grupos se protegen del sol buscando la sombra, evitando salir de las 10 a las 16 horas y usando filtro solar. En contraste, las conductas de riesgo más prevalentes en ambos grupos fueron el realizar actividades domésticas y deportivas al aire libre.

El uso del filtro solar es una de las medidas recomendadas por la OMS para evitar la morbilidad asociada a la exposición a radiación ultravioleta. En nuestro estudio, el $30.6 \%$ de los adolescentes utiliza filtro solar; dato similar a lo encontrado en otros países, como Argentina, en donde el $26 \%$ de los adolescentes lo utilizan de forma cotidiana. ${ }^{6}$ Sin embargo, ese porcentaje es bajo comparado con lo reportado por autores como Castañedo-Cazares y Urasaki, quienes encontraron que más del $20 \%$ se exponían al sol porque consideran saludable broncearse. ${ }^{7,8} \mathrm{En}$ contraste, en adultos observamos que el $48.1 \%$ utilizan filtro solar con regularidad. Esta prevalencia concuerda con lo publicado por Holman en mujeres caucásicas, en donde el $42.6 \%$ se aplicaban un filtro solar habitualmente; aunque la prevalencia en hombres era similar a lo reportado en adolescentes, que en su estudio fue del $18.1 \%{ }^{9}$ Este incremento en la prevalencia del uso del filtro solar en adultos puede atribuirse a que la mayoría de las enfermedades 
desencadenadas por la exposición solar crónica, como cáncer de piel y fotodermatosis, se presentan en adultos; además de que la tolerancia cutánea a la exposición solar intensa e intermitente disminuye con la edad.

Además de la aplicación del filtro solar existen otras medidas de protección que deben promoverse en la población, al ser costo-efectivas. Al respecto, en nuestro estudio (en ambas poblaciones) las conductas más prevalentes fueron el buscar la sombra en un día soleado o evitar el sol de las 10 a las 16 horas; medidas que pueden difundirse en la población, que tendrían un impacto en la morbilidad asociada al sol y que no representarían un costo directo al sistema de salud. El uso de sombrilla tuvo una prevalencia baja, pero fue más frecuente en adultos que en adolescentes, 26.2 versus $10.3 \%$, respectivamente. Al comparar esta medida con otras poblaciones, el uso de sombrilla es una conducta rara, como muestra Şenel al reportar que el $94 \%$ de los jóvenes nunca utilizan sombrilla en un día soleado. ${ }^{10}$

Entre las recomendaciones de protección solar emitidas por la OMS se encuentra el consultar el índice de radiación ultravioleta (IRUV) para planificar las actividades al aire libre. ${ }^{11}$ Aunque dicho índice se puede consultar en el portal de monitoreo atmosférico gubernamental, solo el $3.4 \%$ de los encuestados sabía de su existencia y únicamente el $1.9 \%$ lo consulta de forma cotidiana. Lo anterior muestra que a pesar del esfuerzo gubernamental en la publicación del IRUV, este no es muy conocido ni consultado. Las poblaciones que consultan el índice UV con mayor frecuencia son aquellas en las que el cáncer de piel tiene alta prevalencia, como es el caso de Australia, donde hasta el $93 \%$ de los individuos tiene conocimiento del IRUV, siendo la televisión el principal medio de difusión ( $80 \%) .{ }^{12}$ Algunos estudios demuestran que existe una relación directamente proporcional entre el conocimiento/uso del IRUV y el nivel de desarrollo económico y educativo de un país. ${ }^{12,13} \mathrm{Sin}$ embargo, Sin et al.14 evaluaron la opinión personal y profesional de 400 dermatólogos franceses acerca del índice UV, de los cuales solo el $12.1 \%$ refirieron que lo consultan y llevan a cabo medidas de protección solar basándose en él.

La principal limitación de nuestro estudio fue la forma de medir las conductas relacionadas con el sol, ya que fue mediante autorreporte y no mediante una medición objetiva como el uso de un dosímetro. Sin embargo, en estudios a nivel poblacional se exploran estas conductas por medio de cuestionarios. La otra fuente de sesgo que impacta en la validez externa de nuestros datos es que la muestra no puede considerarse representativa de los adultos o de los adolescentes de la Ciudad de México, ya que los adolescentes pertenecen a una de las preparatorias de la Universidad Nacional Autónoma de México, cuya matrícula corresponde al área geográfica en donde se ubica el plantel y los adultos fueron pacientes que necesitaban atención dermatológica. En esta segunda muestra, las prevalencias de las conductas relacionadas con la exposición solar pueden estar sobreestimando la realidad poblacional, por lo que es necesario incluir preguntas sobre la exposición solar en encuestas de salud nacionales, para obtener resultados que puedan extrapolarse a nuestra realidad.

Finalmente, es necesario puntualizar que con el envejecimiento poblacional de México se espera un incremento en el número de casos de cáncer de piel queratinocítico, ya que su etiología se asocia a la exposición solar prolongada que se correlaciona directamente con la edad. Se propone que las intervenciones para prevenir la morbilidad y mortalidad por exposición solar deben ser multicomponente, es decir, acciones que involucren al individuo, a la comunidad y al gobierno en conjunto. ${ }^{11}$ Dentro de las acciones que deben realizarse desde el sistema de salud están las relacionadas a promover la educación en salud desde la educación básica obligatoria, además de implementar políticas públicas ambientales que promuevan la creación de espacios recreativos con sombra y el uso de ropa de protección en trabajadores al servicio del Estado cuya ocupación sea al aire libre.

\section{Conclusiones}

Conocer las conductas de riesgo y de protección que se llevan a cabo en nuestra población es útil para orientar las intervenciones individuales o a nivel comunitario para disminuir la morbilidad asociada a la exposición solar. La búsqueda de sombra en días soleados y evitar exponerse al sol en el horario de mayor radiación son medidas de prevención que no tienen costo para el individuo, en comparación con el gasto asociado al uso del filtro solar, y que deberían promoverse.

\section{Agradecimientos}

Al M. en C. Eduardo Adolfo Delgadillo Cárdenas, Emily Mayumi Espinosa Ledesma, Gerardo Morales 
Hernández, Iyari Celeste Serrano Vázquez y Alan Ramírez Valverde.

\section{Conflicto de intereses}

Los autores declaran no tener conflicto de intereses alguno.

\section{Financiamiento}

Los autores no recibieron patrocinio para llevar a cabo este artículo.

\section{Responsabilidades éticas}

Protección de personas y animales. Los autores declaran que los procedimientos seguidos se conformaron a las normas éticas del comité de experimentación humana responsable y de acuerdo con la Asociación Médica Mundial y la Declaración de Helsinki.

Confidencialidad de los datos. Los autores declaran que han seguido los protocolos de su centro de trabajo sobre la publicación de datos de pacientes.

Derecho a la privacidad y consentimiento informado. Los autores han obtenido el consentimiento informado de los pacientes y/o sujetos referidos en el artículo. Este documento obra en poder del autor de correspondencia.

\section{Bibliografía}

1. Apalla Z, Lallas A, Sotiriou E, Lazaridou E, Ioannides D. Epidemiological trends in skin cancer. Dermatol Pract Concept. 2017;7(2):1.

2. Skin Cancer [Internet]. American Cancer Society [consultado: 1 julio 2019]. Disponible en: https://www.cancer.org/cancer/skin-cancer.html

3. Radiation: Ultraviolet (UV) radiation and skin cancer [Internet]. World Health Organization; 16 de octubre de 2017 [consultado: 1 julio 2019]. Disponible en: http://www.who.int/uv/faq/skincancer/en/index1.html

4. Seité S, del Marmol V, Moyal D, Friedman AJ. Public primary and secondary skin cancer prevention, perceptions and knowledge: an international cross-sectional survey. J Eur Acad Dermatol Venereol. 2017;31:815-20.

5. Mendoza IA, Pérez DA, Fernando J, Gómez B, Ortega BC, Pablo J, et al. Recomendaciones clínicas para la fotoprotección en México. Dermatología CMQ. 2014;12(4):243-55.

6. Laffarguea AJ, Meredizb J, Bujánb MM, Pierinib AM. Encuesta sobre protección solar en adolescentes deportistas de la Provincia de Buenos Aires. Arch Argent Pediatr. 2011;109(1):30-5.

7. Castañedo-Cazares JP, Torres-Álvarez B, Medellín-Pérez, Aguilar-Hernández GA, Moncada B. Conocimientos y actitudes de la población mexicana con respecto a la radiación solar. Gac Med Mex. 2006;142(6): 451-5.

8. Urasaki MBM, Murad MM, Silva MT, Maekawa TA, Zonta GMA. Exposure and sun protection practices of university students. Rev Bras Enferm. 2016;69(1):114-21.

9. Holman D, Berkowitz Z, Guy G, Hawkins N, Saraiya M, Watson M. Patterns of sunscreen use on the face and other exposed skin among US adults. J Am Acad Dermatol. 2015;73(1):83-92.

10. Senel E, Süslü I. Knowledge, attitudes and behaviors regarding sun protection, effects of the sun, and skin cancer among Turkish high school students and teachers. Dermatol Sin. 2015;33:187-90.

11. Sandhu PK, Elder R, Patel M, Saraiya M, Holman DM, Perna F, et al.; Community Preventive Services Task Force. Community-based interventions to prevent skin cancer: two community guide systematic reviews. Am J Prev Med. 2016;51(4):531-39.

12. Wright $C$, Reeder A, Albers P. School students' knowledge and understanding of the Global Solar Ultraviolet Index. S Afr Med J. 2015;105(12):1024-9.

13. Italia N, Rehfuess E. Is the Global Solar UV Index an effective instrument for promoting sun protection? A systematic review. Health Educ Res. 2012;27(2):200-13.

14. Sin C, Beauchet A, Marchal A, Sigal M, Mahé E. Understanding and use of the global solar UV index ("UV index") by French dermatologists. Ann Dermatol Venereol. 2013;140:15-20. 